http://jmscr.igmpublication.org/home/ ISSN (e)-2347-176x ISSN (p) 2455-0450

crossref DOI: https://dx.doi.org/10.18535/jmscr/v7i12.08

\title{
Role of Contrast MRI in Detection of Breast Lesions and Histopathological Correlation
}

\author{
Authors \\ Dr N. Radha MD (RD)*, Dr Y. Trinadh, Dr Sravya \\ Department of Radio-Diagnosis, Andhra Medical College, Visakhapatnam, India \\ *Corresponding Author \\ Dr N. Radha MD (RD)
}

\begin{abstract}
Background: Breast cancer is the most common cancer in women all over India and accounts for $25 \%$ to $31 \%$ of all cancers in women in Indian cities. No preventive method for breast cancer has yet been defined. Special attention and control can so far only be planned among the known hereditary cancers. Therefore, to improve survival rate early detection is needed. The application of MRI for diagnosis of breast lesions is increasing rapidly. MRI imaging technique that employs time signal intensity curve, obtained by performing MRI scan after injection of contrast agent has emanated as amicable tool for screening of breast cancer, owing to its high sensitivity for detection of abnormalities.

Materials and Methods: Study was conducted at Department of Radio-diagnosis in Andhra Medical College, Visakhapatnam. Study population is 54 patients over duration of 2 years referred from OPD or IPD with clinically palpable breast lesions; non-palpable lesions with inconclusive findings on screening mammogram or screening ultrasonography; and high-risk patients who presented for screening MRI evaluation.

Results: The mean age of the patients was found to be 52.3 years. The range was found to be between 26 to 78 years. 11 patients (Approx. $21 \%$ ) with almost entirely fat type of fibro glandular tissue had malignant lesions. 32 cases i.e. $61 \%$ of patients showed mild background parenchymal enhancement, out of which 24 lesions were found to be malignant and 8 lesions were found to benign. 23 patients (48 \%) had masses with irregular margin and 17 patients (35\%) had masses with speculated margins, 8 cases (17\%) had circumscribed margins.

Conclusion: DCE MRI of breast has been found to be an excellent diagnostic and screening tool for detecting and characterizing breast lesions. Evaluation is done based on morphology and enhancement pattern of the margin and an enhancement pattern of washout. Amongst these two, evaluation of morphology has emerged as the most useful and reliable parameter.
\end{abstract}

\section{Introduction}

The incidence of breast cancer has increased globally over the last several decades. Greatest increase has been observed in Asian countries. Breast cancer is the most common cancer in women all over India and accounts for $25 \%$ to 
$31 \%$ of all cancers in women in Indian cities. The age adjusted rate of breast cancer in Indian women is as high as 25.8 per 100,000 women, with mortality 12.7 per 100,000 women.

The most basic technique of assessment is selfpalpation of breasts and regional lymph nodes, known as breast self-examination (BSE). However, this being a subjective method has its own limitations. In may be difficult to find very small lesions, which can be completely missed on palpation, especially in bulky breasts. However, in the clinical evaluation a thorough palpation of the breasts and regional lymph node stations holds great significance.

Mammography and ultrasonography have been the traditional imaging modalities for evaluation of breast lesions. But the sensitivity of mammography drops to $48 \%$ in high density breasts $^{1}$. Limitations of mammography like incomplete imaging of axillary lymph nodes and blood supply, and underestimation of disease, can be overcome by MRI. The American Cancer Society recommends MRI as an adjunct to mammography for women with lifetime risk of 20-25\% or greater, which include women with strong family history of breast or ovarian malignancy and women who had been treated for Hodgkin's disease ${ }^{2}$.

The advent of MRI has significantly added to the diagnostic armamentarium of the breast conditions that cannot be obtained by other imaging modalities, such as mammography or ultrasound. It has emerged as an important new tool in the fight against breast cancer ${ }^{3}$.

The application of MRI for diagnosis of breast lesions is increasing rapidly. MRI imaging technique that employs time signal intensity curve, obtained by performing MRI scan after injection of contrast agent has emanated as amicable tool for screening of breast cancer, owing to its high sensitivity for detection of abnormalities $^{4,5}$. As recommended by American and European guidelines. Breast MRI is currently the most sensitive detection technique for breast cancer diagnosis ${ }^{6,7}$. It has been stipulated, that in
BRCA1 and BRCA2 mutation carriers, MRI is more sensitive for detecting breast cancers than mammography, ultrasound, or CBE alone ${ }^{8}$.

\section{Materials \& Methods}

It is a Prospective Analytical study. Study was conducted at Department of Radio-diagnosis in Andhra Medical College, Visakhapatnam during February 2017 to October 2018. Study includes 54 patients referred from OPD or IPD with clinically palpable breast lesions; non-palpable lesions with inconclusive findings on screening mammogram or screening ultrasonography; and high-risk patients who presented for screening MRI evaluation.

\section{Inclusion Criteria}

1. Patients aged between 18 to 80 , coming for contrast MRI of lesion for evaluation or screening.

2. Patients who give a valid consent.

\section{Exclusion Criteria}

1. Patients with history of allergy to contrast media.

2. Patients with renal impairment.

3. Non-MRI compatible Cardiac Pacemaker.

4. Non-MRI compatible Metallic Aneurysm clips.

5. Pregnant women.

6. Patients with recurrent breast cancer following chemotherapy or radiotherapy.

\section{Results}

54 patients were studied over duration of 21 months from February 2017 to October 2018.

- Data analysed: 51 patients

- 3 cases were lost to follow-up 
Table 1: Patient Distribution According to Indication of MRI

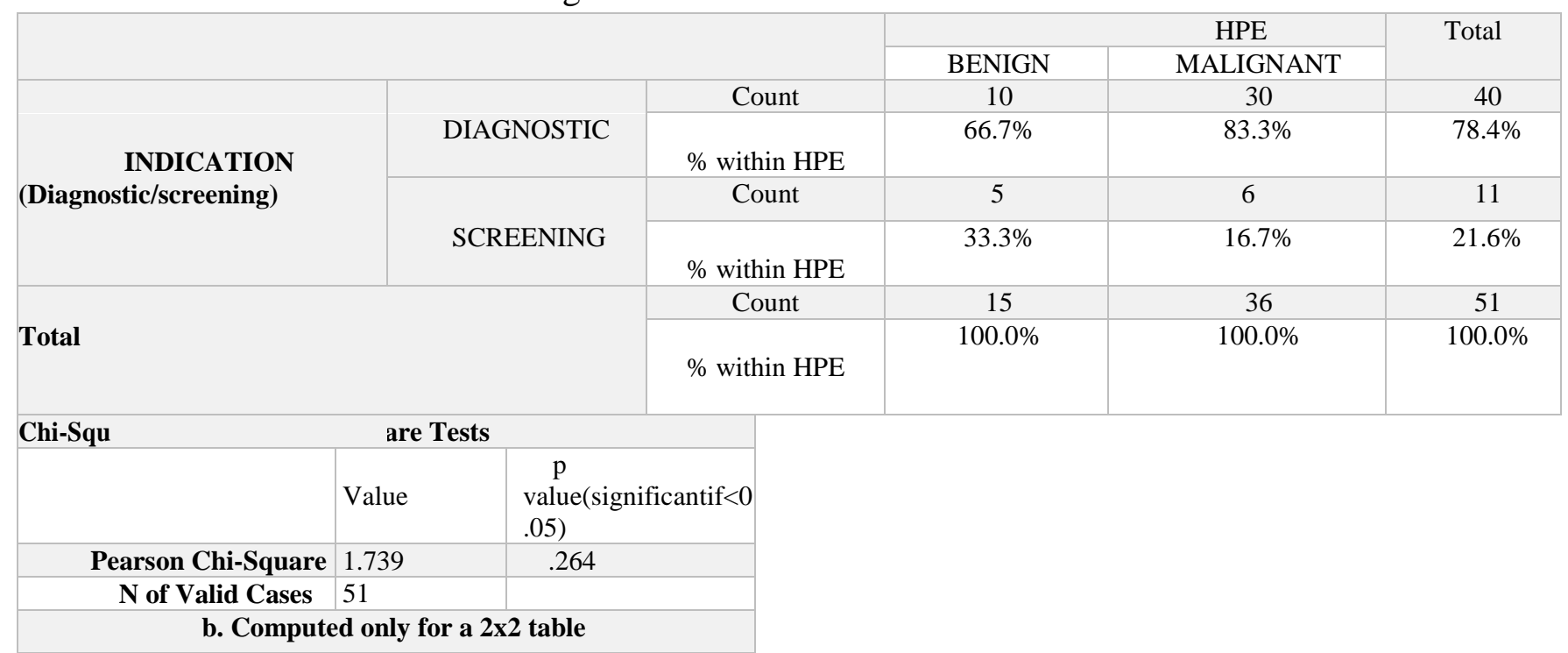

11 patients were a part of screening protocol, whereas 40 patients were evaluated for further diagnostic studies.

11 patients (Approx. $21 \%$ ) with almost entirely fat type of fibro glandular tissue had malignant lesions

41 patients showed symmetrical background enhancement in both breasts and 10 patients showed asymmetric enhancement i.e. different BPE in both breasts.

$>32$ cases i.e. $61 \%$ of patients showed mild background parenchymal enhancement, out of which 24 lesions were found to be malignant and 8 lesions were found to benign.

Table 2: Distribution According to Amount of Fibroglandular Tissue (On MRI) with Histopathology Correlation

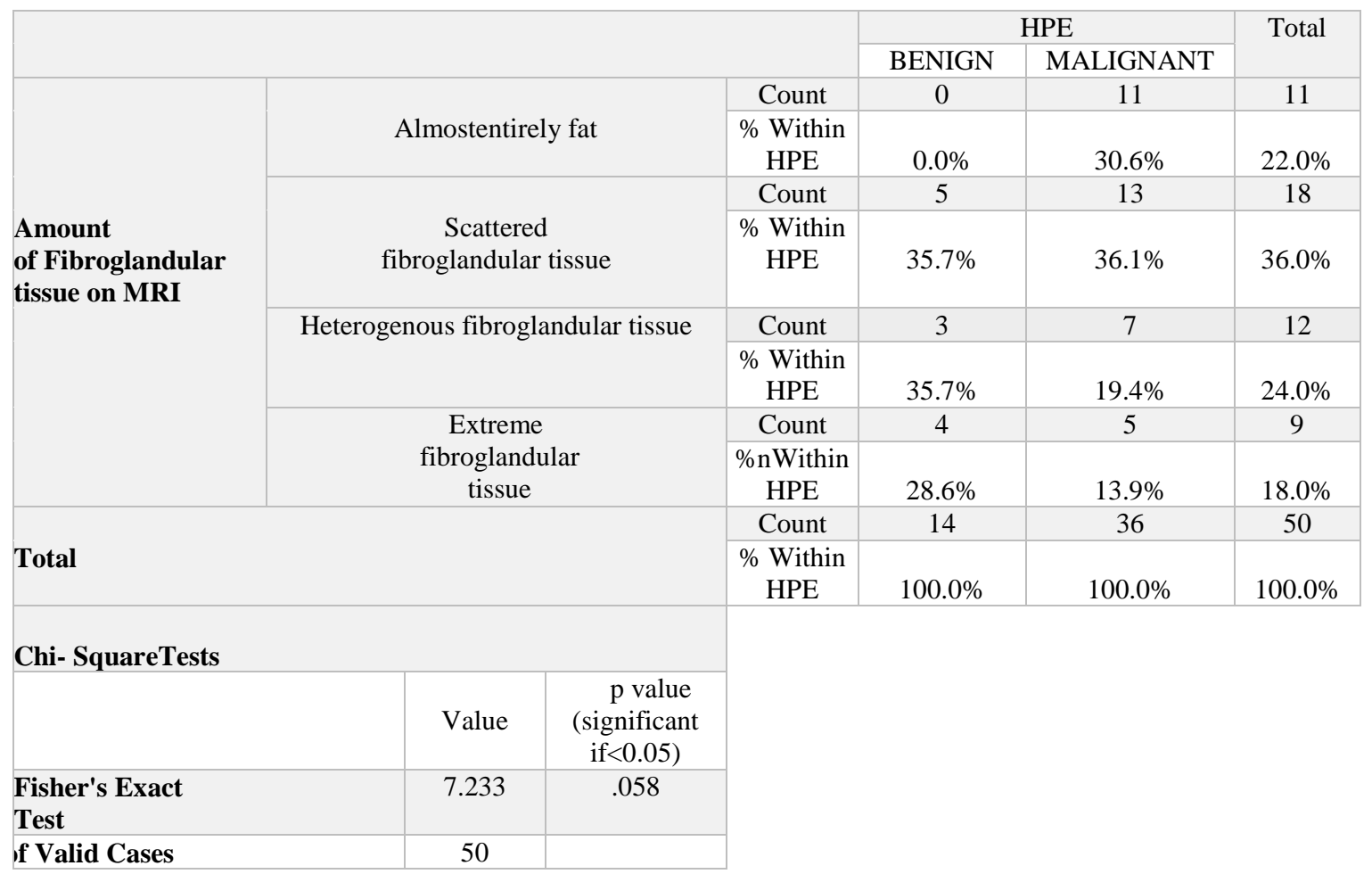


Table 3: Distribution of Mass Shape

\begin{tabular}{|l|c|c|}
\hline MASS SHAPE & DISTRIBUTION & Valid Percent \\
\hline IRREGULAR & 34 & 69.3 \\
\hline OVAL & 8 & 16.3 \\
\hline ROUND & 7 & 14.4 \\
\hline Total & 49 & 100 \\
\hline
\end{tabular}

Table 4: Distribution of Mass Enhancement and Its Correlation to histopathology

\begin{tabular}{|c|c|c|c|c|c|c|}
\hline & & & & & & Total \\
\hline & & & & BENIGN & MALIGNANT & \\
\hline & & TEROGENOUS & Count & 1 & 7 & 8 \\
\hline & & & $\begin{array}{l}\% \text { within } \\
\text { HPE }\end{array}$ & $9.1 \%$ & $20.0 \%$ & $17.4 \%$ \\
\hline & & OMOGENOUS & Count & 6 & 28 & 34 \\
\hline & & & $\begin{array}{l}\% \text { within } \\
\text { HPE }\end{array}$ & $54.5 \%$ & $80.0 \%$ & $73.9 \%$ \\
\hline & & OMOGENOUS & Count & 3 & 0 & 3 \\
\hline MASS ENHANCEMENT & & $\begin{array}{l}\text { ITHIN DARK } \\
\text { SEPTATIONS }\end{array}$ & $\begin{array}{l}\% \text { within } \\
\text { HPE }\end{array}$ & $27.3 \%$ & $0.0 \%$ & $6.5 \%$ \\
\hline & & RING & Count & 1 & 0 & 1 \\
\hline & & IHANCEMENT & $\begin{array}{l}\% \text { within } \\
\text { HPE }\end{array}$ & $9.1 \%$ & $0.0 \%$ & $2.2 \%$ \\
\hline & & & Count & 11 & 35 & 46 \\
\hline & Total & & $\begin{array}{l}\text { \% within } \\
\text { HPE }\end{array}$ & $100.0 \%$ & $100.0 \%$ & $\begin{array}{c}100.0 \\
\%\end{array}$ \\
\hline Chi-Square & & Tests & & & & \\
\hline & Value & $\begin{array}{l}P \text { value (significant } \\
\text { if }<0.05 \text { ) }\end{array}$ & & & & \\
\hline Fisher's Exact Test & 10.968 & .005 & & & & \\
\hline $\mathbf{N}$ of Valid Cases & 46 & & & & & \\
\hline
\end{tabular}

49 cases with mass morphology were evaluated and regrouped into respective categories accordingly Remaining cases showed non mass like enhancement and were assessed separately.

23 patients (48\%) had masses with irregular margin and 17 patients had masses with speculated margins. (35\%) 8 cases $(17 \%)$ had circumscribed margins. Strong correlation was observed. $(\mathrm{p}<0.001)$.

$>28$ patients with homogenous mass enhancement were found to be malignant and 1 benign lesion depicted smooth ring like enhancement.

$59 \%$ of patients showed rapid rise in early phase dynamic contrast, out of which 32 patients were found to be malignant. Estimated $\mathrm{p}$ value was found out to be $<0.001$ suggesting very strong correlation.

$65 \%$ of the lesions showed washout on delayed scan out of which $89 \%$ were found to be malignant on histopathology with estimated $\mathrm{p}$ value to be around $<0.001$ suggesting significant correlation.

$>$ Type III enhancement curve was demonstrated by 30 malignant lesions and 1 benign lesion whereas type I curve was seen in 6 benign lesions.p $<0.001$ suggest significant correlation.

$>14$ out of 16 BIRADS IV and 24 BIRADS $\mathrm{V}$ patients were found to be malignant on histopathology.

$>33 \%$ patients (17) were diagnosed with invasive ductal carcinoma on histopathology lobular carcinoma (12\%) of the total patients and $21 \%$ patients were found to be having ductal carcinoma.

$>10 \%$ patients (5) had fibroadenomas, being the most common benign pathology in our study.

> BIRADS IV and above according to MRI Lexicon were considered to be malignant. 40 lesions (74\%) were diagnosed as malignant and 14 as benign. 


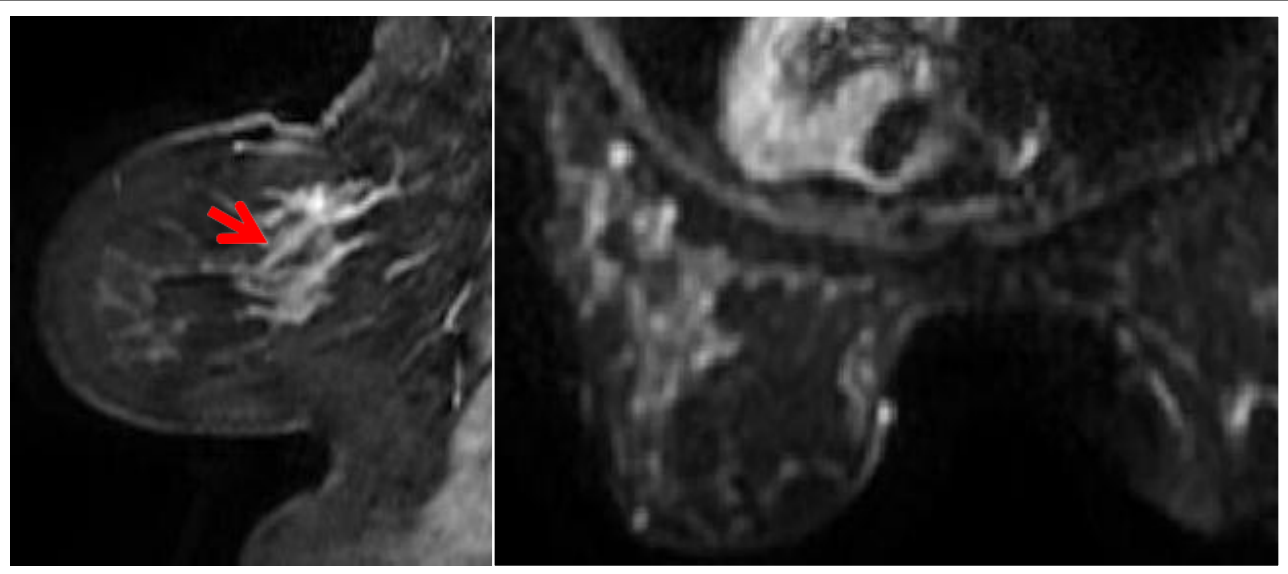

A

B

Figure 1: A Sagittal dynamic post contrast image shows heterogeneous regional non mass like enhancing area in upper half of right breast. B T2 Axial DWI image shows subtle increased intensity in corresponding area.

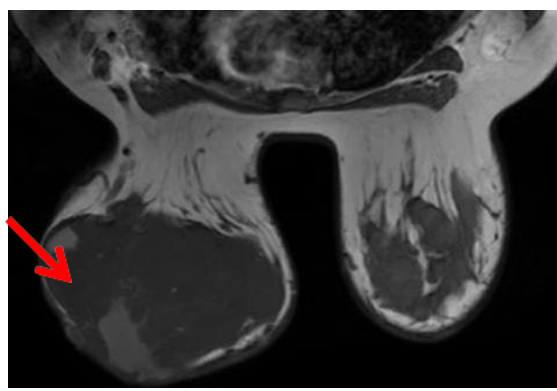

A

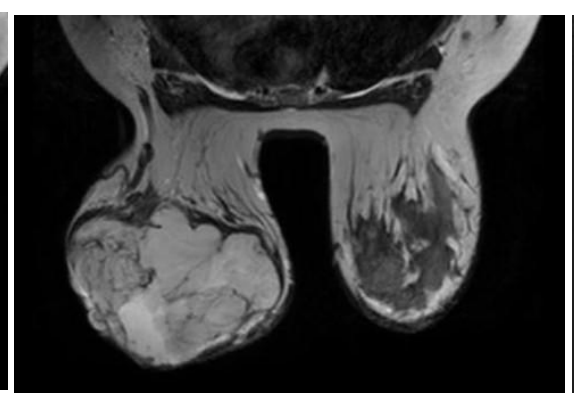

B

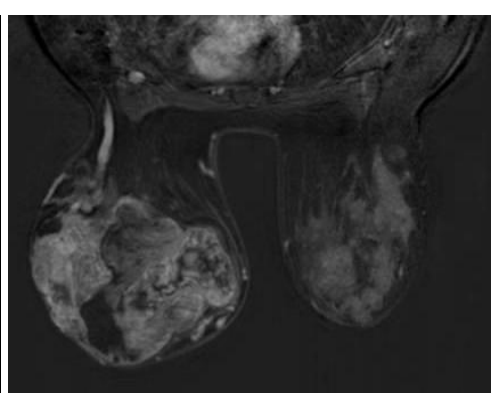

C

Figure 2: A Axial T1WI images. B Axial T2WI.CAxial dynamic contrast enhanced image.

\section{Discussion}

The present study was carried out in 54 cases referred from OPD or IPD of King George Hospital, Visakhapatnam, with clinically palpable breast lesions, non-palpable lesions seen in mammography or ultrasound according to the inclusion and exclusion criteria.

Among the 54 cases 3 were lost to follow up. So, total 51 cases were characterized on dynamic contrast enhancement characteristic and correlated to histopathology. In our study mean age was 52.37 years ranging from 26 years to 78 years. Out of 54 cases, 12 lesions were diagnosed as a part of screening protocol out of which 6 cases had malignant aetiology. Brennan $\mathrm{S}$ et al. ${ }^{9}$ emphasized the importance of screening with breast MRI and detected cancer in $12 \%$ of women with a history of prior breast cancer and in $39 \%$ of women who had biopsies prompted by MRI findings.

\section{Morphological Parameters Fibroglandular Tissue}

In our study, 11 cases had almost entirely fatty type of fibro glandular tissue and all of them were found to have malignant lesions. Whereas, 9 cases had extreme fibro glandular tissue, out of which 5 were found have malignant mass. Fibro glandular tissue type was not found related to lesion detection or pathology of mass in our study.

Similarly, Blumke D et al. ${ }^{10}$ and Brennan et al. ${ }^{9}$ also concluded in their study that MRI performance was not significantly affected by breast density, tumor histology or menopausal status. Sardanelli et al. ${ }^{11}$ in their study, reported that sensitivity for malignancy detection in fatty type of fibro glandular tissue was about $80 \%$ for MRI and the PPV was $65 \%$ (not significant). In breasts with fibro glandular or dense pattern, the sensitivity was $81 \%(\mathrm{p}<0.001)$ and the PPV was $71 \%$ (not significant). 


\section{Background Parenchymal Enhancement}

In our study, 24 malignant lesions showed mild background parenchymal enhancement, 9 malignant lesions showed moderate enhancement and 1 malignant lesion showed marked enhancement. $\mathrm{p}$ value was estimated to be around 0.10 ( $p>0.05)$ and no significant correlation was obtained.

Similar results were demonstrated by Baiti et al. ${ }^{12}$ who assessed background parenchymal enhancement and its correlation with BIRADS in non-high-risk patients and reported that age showed a moderate negative correlation with FGT $(\mathrm{r}=-0.43, \mathrm{p}<0.001)$ and a weak negative correlation with BPE $(\mathrm{r}=-0.28, \mathrm{p}<0.001)$. FGT and BPE correlated moderately $(\mathrm{r}=0.35$, $\mathrm{p}<0.001)$. Final diagnosis of breast cancer displayed very weak negative correlations with FGT $(\mathrm{r}=-0.09, \mathrm{p}=0.046)$ and BPE $(\mathrm{r}=-0.156$, $\mathrm{p}<0.001)$ and weak positive correlation with age $(\mathrm{r}$ $=0.353, \mathrm{p}<0.001)$. On multivariate logistic regression analysis, the only independent covariate for prediction of breast cancer was age (OR 1.032, $\mathrm{p}<0.001)$.

\section{Mass Shape}

In our study, 30 cases had irregular mass shape distribution out of which 28 lesions were found to be malignant with strong correlation. ( $\mathrm{p}=0.01$ ). Remaining 2 cases with irregular mass were found to be benign on histopathology, one being necrotizing inflammation, and the other being fibroadenoma.

Shape of the lesion and its association with malignancy has been studied by Liberman et al. ${ }^{13}$ and similar results were found .According to their study, the feature with the highest PPV was irregular shape (32\% carcinoma) and there was a significantly higher frequency of carcinoma among irregular masses as opposed to lobular shape $(32 \%$ vs. $13 \%, \mathrm{p}=0.17)$. One important point to be noted is that, as per latest ACR BIRADS (5th edition, 2013) ${ }^{14}$, the term lobular has been eliminated from the MRI lexicon , and has been encompassed with oval.

\section{Mass Margin}

In our study, masses in 23 cases were found to have irregular margins, out of which 19 were malignant. 17 cases had mass with spiculated margins, all of which were found to be malignant on histopathology, and a strong correlation was observed; $p$ value was estimated to be $<0.001$.

Liberman et al. ${ }^{13}$ also concluded the same, with significantly higher frequency of malignancy in masses with spiculated margins $(80 \%$ vs $20 \%$, p $=$ $0.01)$. In their study, they found that carcinoma was present in $22 \%$ of masses with irregular margins and in $17 \%$ of smoothly marginated masses.

7 out of 8 lesions with circumscribed margins, in our study, were found to be benign on histopathology.1 case of malignant mass, diagnosed as mucinous carcinoma on histopathology was found to have circumscribed margins.

Nunes et al. ${ }^{15}$ also concluded in their previous studies that the MR imaging features with the highest PPV were spiculated margin (77-95\% cancer) and smooth borders were associated with benignity in $97-100 \%$ of cases in their prior reports.

\section{Mass Enhancement Characteristics}

In our study, 34 cases showed homogenous enhancement and 8 showed heterogeneous enhancement out of which 28 and 7 were found to be malignant on histopathology, respectively.

3 cases showing homogenous enhancement with dark septations, were found to be fibroadenoma on histopathology.1 case showed peripheral rim enhancement, which on histopathology, was found to be an abscess.

Out of 5 cases with non-mass like enhancement patterns, regional non-mass like enhancement was seen in 3 cases with biopsy proven fibrocystic breast disease. Diffuse heterogeneous and homogenous pattern was seen in 2 cases which turned out to be lobular carcinoma in-situ on biopsy. 
Nunes et al. ${ }^{15}$ and Liberman et al. ${ }^{13}$ concluded that irregular shape and rim enhancement were associated with a higher frequency of carcinoma than other shape and enhancement features. These differences, however, did not achieve statistical significance.

\section{Dynamics of Contrast Enhancement}

The two components of time intensity curve, i.e. initial phase kinetics and delayed phase kinetics were evaluated.

Initial phase kinetics was regrouped into slow, medium and rapid rise. Rapid rise was found in 30 lesions (59\%), all of which turned out to be malignant on histopathology. $\mathrm{p}<0.001$ signified good correlation.

Medium rise was found in $16(31.4 \%)$ cases, out of which $11(73.3 \%)$ were benign and $5(13.99 \%)$ were malignant on histopathology.

Slow rise was found in $5(9.8 \%)$ cases, out of which $4(26.7 \%) 4$ were proven to be benign and $1(2.8 \%)$ was found to be malignant on histopathology.

The most common type of pattern observed in delayed phase kinetics in our study was rapid washout pattern amounting to $64.7 \%$ of all the cases. Persistent and plateau types of delayed enhancement was seen in 8 and 11 cases respectively.

Rapid washout was seen in 32 malignant lesions and 1 benign lesion with strong correlation. $(\mathrm{p}<0.001)$.

33 cases (61\% approximately) demonstrated Type III Kuhl's curve (Time Intensity curve/ enhancement curve), 14 cases exhibited Type II and 7 cases showed Type I enhancement curve patterns.

Out of the 33 cases with Type III curve, 30 were found to be malignant on histopathology with significant correlation $(\mathrm{p}<0.001) .2$ cases were lost to follow up and 1 case was found to be fibroadenoma on histopathology.

Houserkova et al. ${ }^{16}$ also evaluated the level of enhancement and found slow initial peak in $6 \%$ enhanced DCIS, medium peak in $27 \%$ and rapid peak in $67 \%$ of them. In only $3 \%$ case with lowgrade DCIS persistent enhancement pattern was found. In delayed phase, $67 \%$ of the DCIS patients were found to have plateau enhancement pattern, and in $30 \%$ DCIS cases there was washout enhancement pattern.

\section{Lesion Histology and Types}

Out of 51 lesions evaluated in our study, the most common lesion identified was invasive ductal carcinoma (33\%; 17). 11 cases $(21.6 \%)$ had ductal carcinoma in-situ on histology.

Similar results and prevalence of lesions were demonstrated by Kinkel $\mathrm{K}$ et al. ${ }^{17}$ who evaluated dynamic contrast MRI in breast lesions and found 12 invasive, 3 ductal carcinomas in situ and 19 mixed cancer in their study group.

In our study, amongst benign lesions, fibrocystic breast disease (total number 6) and fibroadenoma (total number 5) were the most common lesions.

1 abscess, 1benign phyllodes, 1fibroadenomatosis and 1 case of bilateral papilloma were identified.

\section{Conclusion}

In conclusion, DCE MRI of breast has been found to be an excellent diagnostic and screening tool for detecting and characterizing breast lesions. Evaluation is done based on morphology and enhancement pattern of the margin and an enhancement pattern of washout. Amongst these two, evaluation of morphology has emerged as the most useful and reliable parameter.

Kinetic curve assessment, however, adds significantly in increasing the sensitivity of this modality. Imaging parameters for the characterization of suspicious breast lesions were also assessed. This modality proved to have excellent histopathology correlation.

The following salient features have been concluded by our study:

- Breast MRI finding are impendent of the composition of fibro glandular parenchyma. 
- Type III curve is most frequently associated with malignancy $(61 \%)$ in our study.

- Most of the malignant masses are associated with rapid enhancement

- Presence of internal non enhancing septations is almost always suggestive of fibroadenoma.

- Lobular carcinomas often show a nonmass like enhancement.

- Malignancies that are occult on Mammogram or ultrasonography can be picked up on DCE MRI

- DCE MRI proves to be a very useful tool in screening of high-risk cases

With the help of a multimodality approach, making use of DCE MRI along with ultrasonography and mammography, we can go a long way in screening and early detection of breast lesions.

In fact, with a sensitivity of as high as $100 \%$ (as in our study) DCE MRI breast holds the potential to alleviate the need of a biopsy which is an invasive procedure.

\section{References}

1. Joe BN, Sickles EA. The evolution of breast imaging: past to present. Radiology. 2014 Nov;273(2 Suppl):S23-44.

2. Aggrawal G, Su MY, NalciogluO, Feig, SA, Chen JH Significance of breast lesion descriptors in the ACR BI-RADS MRI lexicon. Cancer. 2009 April 115(7): 13631380.

3. Ian Gardiner, MD, UrveKuusk, MD, FRCPC, Bruce B. Forster, MD, MSc, FRCPC, Audrey Spielmann, MD, FRCPC. Breast magnetic resonance imaging. BCMJ, Vol. 47, No. 10, December, 2005, page(s) 543-548.

4. Kuhl CK, Mielcareck P, Klaschik S, Leutner C, Wardelmann E, Gieseke J, Schild HH. Dynamic breast MR imaging: are signal intensity time course data useful for differential diagnosis of enhancing lesions? Radiology. 1999 Apr;211(1):10110.

5. Schnall MD, Rosten S, Englander S, Orel SG, Nunes LW. A combined architectural and kinetic interpretation model for breast MR images. Acad Radiol. 2001 Jul;8(7):591-7.

6. Saslow D, Boetes C, Burke W, Harms S, Leach MO, Lehman CD, Morris E, Pisano E, Schnall M, Sener S, Smith RA, Warner E, Yaffe M, Andrews KS, Russell CA; American Cancer Society Breast Cancer Advisory Group. American Cancer Society guidelines for breast screening with MRI as an adjunct to mammography. CA Cancer J Clin.2007 Mar-Apr;57(2):75-89. Erratum in: CA Cancer J Clin. 2007 MayJun;57(3):185.

7. Mann RM, Kuhl CK, Kinkel K, Boetes C. Breast MRI: guidelines from the European Society of Breast Imaging. Eur Radiol. 2008 Jul;18(7):1307-18.

8. Warner E, Plewes DB, Hill KA, Causer PA, Zubovits JT, Jong RA, Cutrara MR, DeBoer G, Yaffe MJ, Messner SJ, Meschino WS, Piron CA, Narod SA. Surveillance of BRCA1 and BRCA2 mutation carriers with magnetic resonance imaging, ultrasound, mammography, and clinical breast examination. JAMA. 2004 Sep 15;292(11):1317-25.

9. D’Orsi CJ, Sickles EA, Mendelson EB, Morris EA, et al. ACR BI-RADS® Atlas, Breast Imaging Reporting and Data System. Reston, VA, American College of Radiology; 2013

10. Rausch DR, Hendrick RE. How to optimize clinical breast MR imaging practices and techniques on your 1.5-T system. Radiographics. 2006 SepOct;26(5):1469-84.

11. Grimm LJ, Anderson AL, Baker JA, Johnson KS, Walsh R, Yoon SC, Ghate $\mathrm{SV}$. Interobserver Variability Between Breast Imagers Using the Fifth Edition of 
the BI-RADS MRI Lexicon. AJR Am J

Roentgenol. 2015 May;204(5):1120-4.

12. O'Connor JP, Tofts PS, Miles KA, Parkes LM, Thompson G, Jackson A. Dynamic contrast- enhanced imaging techniques: CT and MRI. Br J Radiol. 2011 Dec;84 Spec No 2:S112-20.

13. King V, Brooks JD, Bernstein JL, Reiner AS, Pike MC, Morris EA. Background parenchymal enhancement at breast MR imaging and breast cancer risk. Radiology $2011 \mathrm{Jul}$;260(1):50-60.

14. Telegrafo M, Rella L, Stabile Ianora AA, Angelelli G, Moschetta M. Breast MRI background parenchymal enhancement (BPE) correlates with the risk of breast cancer. MagnReson Imaging. 2016 Feb;34(2):173-6.

15. Bennani-Baiti B, Dietzel M, Baltzer PA. Correction: MRI Background Parenchymal Enhancement Is Not Associated with Breast Cancer. PLoS One. 2016 Sep 8;11(9):e0162936.

16. Flickinger FW, Allison JD, Sherry RM, Wright JC. Differentiation of benign from malignant breast masses by time-intensity evaluation of contrast enhanced MRI. MagnReson Imaging. 1993;11(5):617-20.

17. Nunes LW, Schnall MD, Siegelman ES, Langlotz CP, Orel SG, Sullivan D, Muenz LA, Reynolds CA, Torosian MH. Diagnostic performance characteristics of architectural features revealed by high spatial-resolution MR imaging of the breast. AJR Am J Roentgenol. 1997 Aug; 169(2):409-15. 